\title{
Recurrent Neurothekeoma of the Foot
}

\author{
Mehtap Karamese ${ }^{1}$, Mustafa Hanci ${ }^{2}$, Hasan Esen $^{2}$, Mustafa Keskin ${ }^{2}$ \\ ${ }^{1}$ Plastic Reconstructive and Aesthetic Surgery Department, Selcuklu Faculty of Medicine, Selcuk University, Konya, Turkey; ${ }^{2}$ Plastic \\ Reconstructive and Aesthetic Surgery Department, Meram Faculty of Medicine, Selcuk University, Konya, Turkey. \\ Email: \{mehtapef, yeahop\}@yahoo.com,drmkeskin@hotmail.com
}

Received April $4^{\text {th }}, 2012$; revised May $9^{\text {th }}, 2012$; accepted June $6^{\text {th }}, 2012$

\begin{abstract}
Nerve sheath myxomas (NEUROTHEKEOMA) are uncommon tumors of nerve sheet origin. These tumors most frequently arise from in the dermis and subcutaneous tissues of the head and neck, and upper extremities. Neurothekeoma may be either asymptomatic or may present as a painful raised, skin colored, well circumscribed dermal lesion averaging $1 \mathrm{~cm}$ in diameter. This entity is especially rare in the foot and we present a 15 years old male patient with a large $(6.5 \mathrm{~cm})$ recurrent eurothekeoma of the left foot.
\end{abstract}

Keywords: Foot; Neurothekeoma; Recurrent

\section{Introduction}

Neurothekeomas are rare, benign soft tissue tumors that are considered to be of nevre sheath origin. These tumors are present most commonly on the head and neck and upper extremities and may cause significant local destruction of cosmetically sensitive areas [1]. Surgical excision has been advocated as the treatment of choice, but long-term follow-up data are not available regarding recurrence rates [2]. Neurothekeomas characteristically arise from the reticular dermis in children and young adults, usually female with a mean age of 21.6 years [3]. These are typically slow growing, painless lesions that are less than $3 \mathrm{~cm}$ in diameter. We report neurothekeoma of the foot with diameter of $5 \mathrm{~cm}$.

\section{Case}

A 15-year-old male patient, with a recurrent, slowly progressing protuberant lesion of left leg was referred to our clinic from our colleagues in vascular surgery. In his history, patient has undergone a surgery in an orthopedic surgery clinic in 2007 and histological examination of this excision specimen revealed a nodular lesion with a loose mixoid stroma including multiloculated lipoblasts and mature liposites and also dilated capillary vessels. The lump recurred within one year and patient has applied vascular surgery department where MR imaging was performed. Then the patient referred to our clinic with what was thought to be vascular malformation due to his MR imaging. We performed a MR Angiography and its reported that the lesion was not compatible with the vascular malformation or higroma and histological examination was advised. On physical examination a painless, slow growing, soft mass which was composed of four lobules extending lateral surface of foot. This mass was $5 \mathrm{~cm}$ diameter. There was no popliteal or inguinal lymphadenopathy. The patient was operated on under general anesthesia. We thought to perform wide excision of the recurrent lesion and during surgical exploration we found that the lesion was strictly adherent to subcutaneous tissue beneath the skin and to the underlying bone tissue. The lesion was excised with a deep margin, and the defect was covered with a full thickness skin graft (FTSG). Wound healed within 2 weeks and there was no early postoperative evidence of recurrence or wound complication during the follow up period of 18 months (Figures 1-3). Histopathological investigation of the tumor was reported as a nerve sheet myxoma (neurothekeoma). Microscopically the neoplasm showed a broad (dermis-hypodermis), spindle and elliptic shaped normocromatic cells arranged in fascicles disposed at least in a lobulated manner in a mixoid stroma, without atypical mitotic activity, and immunohistochemically alcian blue staining performed, S-100(+), GFAP $(+)$, CD34(+), ACTIN(+) (Figure 4).

\section{Discussion}

Neurothekeoma is a term introduced by Gallager and Helwig in 1980 to describe a superficial tumor of purported nerve sheath derivation [4]. Traditionally, this tumor has been subclassified as cellular, mixed, or myxoid, depending on the amount of myxoid matrix [5]. Neurothekeomas are morphologically and immunohistochemically distinct from true nevre sheath myxomas. An 


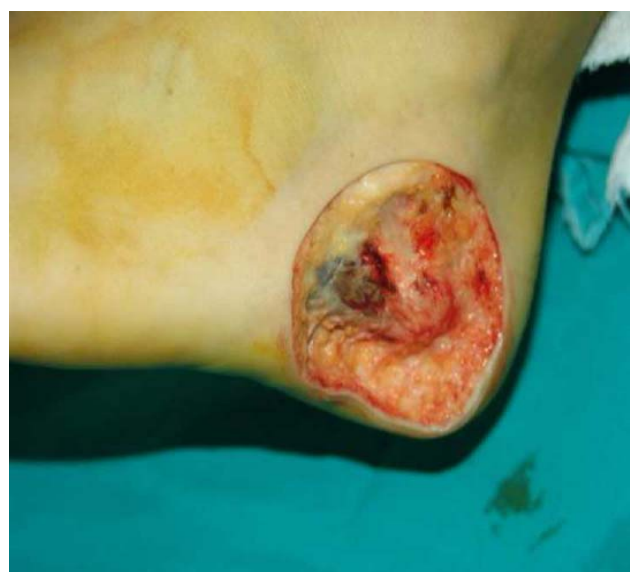

Figure 1. Left foot lateral view: intraoperative.

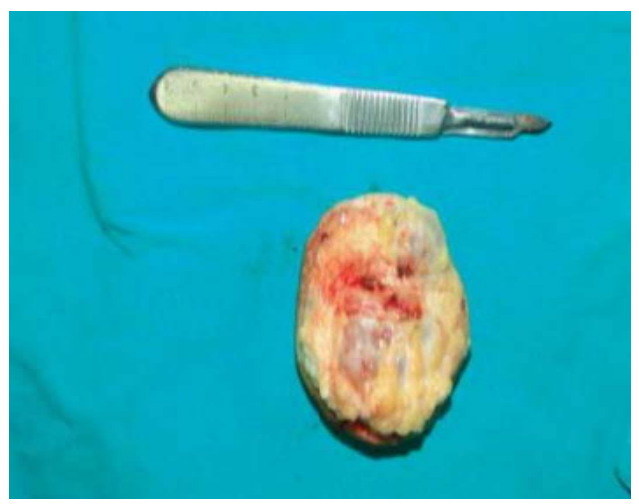

Figure 2. Surgical specimen.

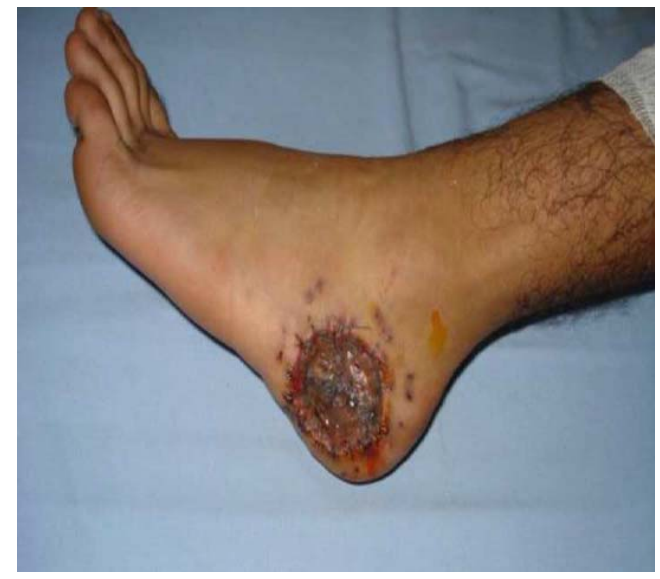

Figure 3. Left foot lateral view: early postoperative.

origin from fibroblastic cells with the ability to differentiate into myofibroblasts and a tendency to recruit histiocytic cells is postulated. It is generally accepted that myxoid neurothekeoma is a tumor of neural origin and cellular neurothekeoma is a tumor of neuroectodermal origin. However, the histogenesis of cellular neurothekeoma has not yet been fully elucidated, the tumor cells of myxoid neurothekeoma were shown to be S-100positive; however, those of the cellular neurothekeoma

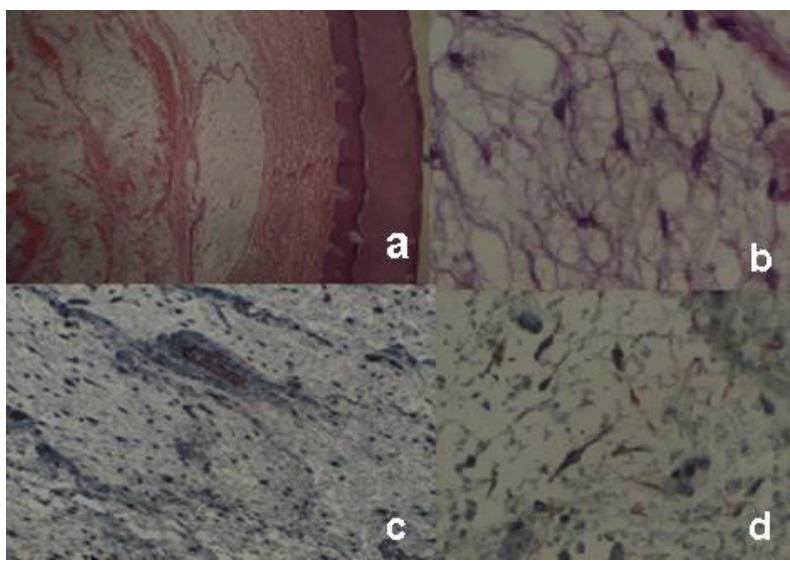

Figure 4. Pathological images. (a) Beneath the skin, cells with well demarcated margins and myxoid stroma (Hematoxylin \& Eosin $\times 40$ ); (b) Spindle shaped fibriller extensions of these cells (Hematoksilen \& Eozin $\times 400$ ); (c) Immunohistochemically actin negative $(\times 100)$; (d) Glial fibrillary acidic protein $($ GFAP) pozitive $(\times 200)$.

were S-100-negative. Myxoid more common onthe trunk and lower extremities and cellular are more common on the head and neck and upper extremities [6]. Neurothekeoma shows a clear female predominance. Neurothekeoma may be either asymptomatic or may present as a painful raised, skin colored, well-circumscribed dermal lesion averaging $1 \mathrm{~cm}$ in diameter that involve the skin and superficial subcutis [7]. Deep involvement of the subcutis is uncommon, and skeletal muscle involvement is rare and largely restricted to the facial region. In Gallager and Helwig's series, the mean diameter of neurothekeomas was $1 \mathrm{~cm}$; ranging between 0.4 and 1.8 $\mathrm{cm}$. But our case was huge and recurrent tumor [8]. The presented case shows a less common location for this rare benign tumor of putative neural origin and it had about $5 \mathrm{~cm}$ diameter. Also this was recurrent tumor. We did not attempt aggressive treatment. Local oncology committee did not recommend the other treatment modalities like chemotherapy or radiotherapy. In the literature Fetsch said that there is an overestimation of the true recurrence rate. Regrowth or local recurrences are seen in about 7 percent of patients [2,9]. The rare cases of local recurrence may be attributed to either incomplete excision or to a new tumor in the tissue. We performed wide excision. We know there are long follow up period like that 10 years in the literature. Clinically, a multitude of differential diagnoses exist for neurothekeoma, including fibroma, dermatofibroma, leiomyoma, neurilemomma, neurofibroma, higroma and hemangioma. Cellular neurothekeoma was described by Barnhill and Mihm in 1990 [10]. Cellular neurothekeoma must be carefully differentiated from melanoma, especially some neurotropic subtypes. Noting positive S100 immunoreactivity in myxoid neurothekeoma and a lack of S100 expression in 
cellular neurothekeoma are suggested distinct origins or divergent differentiation. Although NT is rare in the lower extremity it is important to consider this entity in the differential diagnoses, as it is indispensable to distinguish it from malignant lesions so that aggressive therapy could be avoided. Excision with clear margins is the choice of treatment. These tumors are reported to be confused with malignant tumors because of their pathological findings. In neurothekeomas, treatment with local excision would be sufficient. Even with incomplete excision, they have a low recurrence rate and carry a good prognosis. There is no sign in the literatüre to malign transformation of neurothekeoma. According to Fetsch the present data and past studies are insufficient, to make a definite statement regarding the clinical significance of the atypical features noted in this report (i.e., increased nuclear atypia, an elevated mitotic count, and atypical mitotic figures). We are not aware of a neurothekeoma with atypical features having behaved in a low-grade malignant fashion, but we cannot definitely rule out such a possibility. It is clear from the very low metastatic rates of some "borderline" soft tissue tumors that large numbers of cases with long-term follow-up are needed to identify the infrequent example with an adverse outcome. This is especially true when the majority of examples are small at presentation and when they have a predilection for the skin [2].

\section{Conclusion}

In summary, we have presented the clinical, histopathologic, and immunohistochemical findings for recurrent neurothekeoma example. Neurothekeomas have a peak incidence in the second decade of life; a female predominance; a strong predilection for the head, upper extremities and shoulder girdles; and a low local recurrence rate. Atypical examples of neurothekeoma are underrepresented in the literature, and their clinical significance remains unclear. These latter tumors require further study; however, complete excision with follow-up should prove adequate in most, if not all, instances.

\section{REFERENCES}

[1] D. J. Koumanis and L. T. Glickman, "Facial Neuro- thekeoma in a 10-Year-Old Child," Canadian Journal of Plastic Surgery, Vol. 15, No. 3, 2007, pp. 175-177.

[2] J. F. Fetsch, et al., "Neurothekeoma: An Analysis of 178 Tumors with Detailed İmmunohistochemical Data and Long-Term Patient Followup İnformation," The American Journal of Surgical Pathology, Vol. 31, No. 7, 2007, pp. 1103-1114. doi:10.1097/PAS.0b013e31802d96af

[3] K. D. Mertz, et al., "A Rare Case of Atypical Cellular Neurothekeomain a 68-Year-Old Women," Journal of Cutaneous Pathology, Vol. 36, No. 11, 2009, pp. 12101214. doi:10.1111/j.1600-0560.2009.01261.x

[4] R. L. Gallager and E. B. Helwig, "Neurothekeoma-A Benign Cutaneous Tumor of Neural Origin," American Journal of Clinical Pathology, Vol. 74, No. 6, 1980, pp. 759-764.

[5] U. Wollina, et al., "Myxoid Neurothekeoma-A Painful Case in a Less Common Location," Dermatology Online Journal, Vol. 15, No. 4, 2009, p. 3.

[6] J. F. Fetsch, W. B. Laskin and M. Miettinen, "Nerve Sheath Myxoma: A Clinicopathologic and Immunohistochemical Analysis of 57 Morphologically Distinctive, S-100 Protein- and GFAP-Positive, Myxoid Peripheral Nerve Sheath Tumors With a Predilection for the Extremities and a High Local Recurrence Rate," The American Journal of Surgical Pathology, Vol. 29, No. 12, 2005, pp. 1615-1625.

[7] S. Avninder, V. Ramesh and S. Vermani, "Benign Nerve Sheath Myxoma (Myxoid Neurothekeoma) in the Leg," Dermatology Online Journal, Vol. 13, No. 2, 2007, p. 14.

[8] R. L. Gallager and E. B. Helwig, "Neurothekeoma-A Benign Cutaneous Tumor of Neural Origin," American Journal of Clinical Pathology, Vol. 74, No. 6, 1980, pp. 759-764.

[9] J. L. Hornick and C. D. Fletcher, "Cellular Neurothekeoma: Detailed Characterization in a Series of 133 Cases," The American Journal of Surgical Pathology, Vol. 31, No. 3, 2007, pp. 329-340. doi:10.1097/01.pas.0000213360.03133.89

[10] R. L. Barnhill, et al., "Cellular Neurothekeoma: A Distinctive Variant of Neurothekeoma Mimicking Nevomelanocytic Tumors," The American Journal of Surgical Pathology, Vol. 14, No. 2, 1990, pp. 113-120. doi:10.1097/00000478-199002000-00002 